Original article

\title{
The inflammatory potential of diet is related to incident frailty and slow walking in older adults
}

\author{
Q7 Martin Laclaustra a, b, * , Fernando Rodriguez-Artalejo ${ }^{\text {b, c }}$, Pilar Guallar-Castillon b, c, \\ Jose R. Banegas ${ }^{\text {b }}$, Auxiliadora Graciani ${ }^{\text {b }}$, Esther Garcia-Esquinas ${ }^{\text {b }}$, Esther Lopez-Garcia b, c \\ a Instituto de Investigación Sanitaria de Aragón (IIS Aragón), CIBERCV, Agencia Aragonesa para la Investigación y el Desarrollo (ARAID), Translational \\ 01 Research Unit, Hospital Universitario Miguel Servet, Paseo Isabel la Católica, 1-3, 50009, Zaragoza, Spain \\ ${ }^{\mathrm{b}}$ CIBERESP and Department of Preventive Medicine and Public Health, School of Medicine, Universidad Autónoma de Madrid/Idipaz, Arzobispo Morcillo, 4, \\ 28029, Madrid, Spain \\ ${ }^{\mathrm{c}}$ IMDEA-Food Institute, CEI UAM+CSIC, Madrid, Spain
}

\section{A R T I C L E I N F O}

\section{Article history:}

Received 21 December 2017

Accepted 13 January 2019

\section{Keywords:}

Frailty

Dietary patterns

Inflammation

Disability

Cohort

\section{S U M M A R Y}

Background: Certain foods and dietary patterns have been associated with both inflammation and frailty. As chronic inflammation may play a role in frailty and disability, we examined the association of the inflammatory potential of diet with these outcomes.

Methods: Data were taken from 1948 community-dwelling individuals $\geq 60$ years old from the SeniorsENRICA cohort, who were recruited in 2008-2010 and followed-up through 2012. Baseline diet data, obtained with a validated diet history, was used to calculate Shivappa's Dietary Inflammatory Index (DII), an "a priori" pattern score which is based on known associations of foods and nutrients with inflammation, and Tabung's Empirical Dietary Inflammatory Index (EDII), an "a posteriori" pattern score which was statistically derived from an epidemiological study. At follow-up, incident frailty was assessed with Fried's criteria, and incident limitation in instrumental activities of daily living (IADL) with the LawtonBrody index. Statistical analyses were performed with logistic regression, and adjusted for the main confounders.

Results: Compared with individuals in the lowest tertile of DII, those in the highest tertile showed higher risk of frailty (odds ratio [OR] 2.48; 95\% confidence interval $[\mathrm{CI}]: 1.42,4.44$, p-trend $=0.001$ ) and IADL disability (OR: 1.96; 95\% CI: 1.03, 3.86, p-trend $=0.035$ ). By contrast, EDII did not show an association with these outcomes. The DII score was associated with slow gait speed, both as a low score in the Short Physical Performance Battery test (OR: 1.82; 95\% CI: 1.27, 2.62, p-trend $=0.001$ ) and as a positive Fried's criterion (OR: 1.64; 95\% CI: 1.08, 2.51, p-trend $=0.021$ ), which use different thresholds.

Conclusions: DII predicted frailty and IADL while EDII did not. DII is able to measure diet healthiness in terms of physical decline in addition to avoidance of inflammation.

Registered on: ClinicalTrials.gov number, NCT01133093.

@ 2019 Published by Elsevier Ltd.

\section{Introduction}

Aging is associated with progressive limitations in physical functioning and with disability. The frailty syndrome [1] is a criteria-based condition which heralds the disability process, and identifies older adults with increased risk of death, hospitalization and falls [2]. Lifestyle, in particular physical activity and diet, has

\footnotetext{
* Corresponding author. Translational Research Unit (IIS Aragón), Hospital Universitario Miguel Servet, Paseo Isabel la Católica, 1-3, 50009, Zaragoza, Spain.

E-mail address: martin.laclaustra@unizar.es (M. Laclaustra).
}

strong influence on the aging process [3]. In particular, diet [4], studied as specific foods and nutrients $[4,5]$ or as patterns [6-8] has been associated with the risk of frailty and disability in older adults.

Chronic inflammation may play a role in the pathophysiology of frailty, although further research should still clarify the actual mechanisms [9]. Shivappa et al. proposed the dietary inflammatory index (DII), which captures a priori-defined dietary patterns linked to higher inflammatory markers, based on published evidence [10] and thus can be used to link diet and inflammation. Very recently, an empirical (a posteriori) dietary inflammatory index (EDII) has been developed using prospective data from the large Nurses' 
Health Study [11]. DII has demonstrated association with several diseases, including those leading world mortality and morbidity: cardiovascular diseases [12,13], which share risk factors with frailty, and cancer [14], but also metabolic syndrome [15] and depression [16] among others. Only a previous article has examined the association between an inflammatory index and the risk of frailty [17] and none has assessed the association with disability. As both indices differ in the way they were developed it is possible that their information is not equivalent and it would be worth analyzing the association of both with aging-related outcomes.

Thus, we hypothesized that inflammatory dietary patterns are associated with higher risk of frailty and disability, and we tested this hypothesis with data from the Seniors-ENRICA cohort of older adults in Spain.

\section{Methods}

\subsection{Study design and participants}

The Seniors-ENRICA study is a cohort of 2614 communitydwelling individuals aged 60 years or older, who were recruited between 2008 and 2010 and have been followed-up through 2012 [18]. Among survivors $(n=2519)$, we excluded 9 participants with dementia or Alzheimer disease at baseline, 12 with missing data on diet, and 550 on questionnaires or function tests, for a final sample size of 1948 (See Flowchart). At baseline, physical and laboratory examinations, a frailty assessment, and several health questionnaires, including a diet history and a Lawton and Brody scale, were performed. At follow-up, the same procedures were repeated with the addition of some others like the Short Physical Performance Battery (SPPB). This study describes the longitudinal association of baseline dietary inflammatory indices with aging-related outcomes: a reduced physical performance, frailty, and disability. Specific exclusions to select participants at risk for each analysis are described below, in the statistical analyses section (see Flowchart). Informed consent in writing was obtained from all participants, and the study was approved by the Clinical Research Ethics Committee of the La Paz University Hospital in Madrid.

\subsection{Exposure measurements: diet and inflammatory dietary indices}

A computer-based diet history was used to collect the participant's regular diet. The interview collected information from 880 different foods and the nutrient intake from them is calculated using Spanish food composition tables. This diet history is validated [19] and provides an estimate in daily grams of foods and nutrients that represents the average intake during a year.

To assess the inflammatory components of diet, we used the DII and EDII. The DII was calculated according to Shivappa's procedure [10]. In brief, 32 (see list on appendix) of the possible 45 items (daily amounts of food or nutrients) were normalized using the world averages and standard deviations provided in the procedure [10]. Those values were then converted to centered percentile scores that were subsequently multiplied by each provided parameter factor ("food parameter effect score") [10]. The sum of the 32 values resulted in a DII score for each participant. A higher DII score indicates a greater diet's inflammatory component (see Fig. 1).

EDII calculation was based on 18 food groups (see list on appendix), ascertained as daily portions [11]. We summed daily intake of foods in each of the 18 groups, and then converted the values to portions by dividing them for a group-specific portion

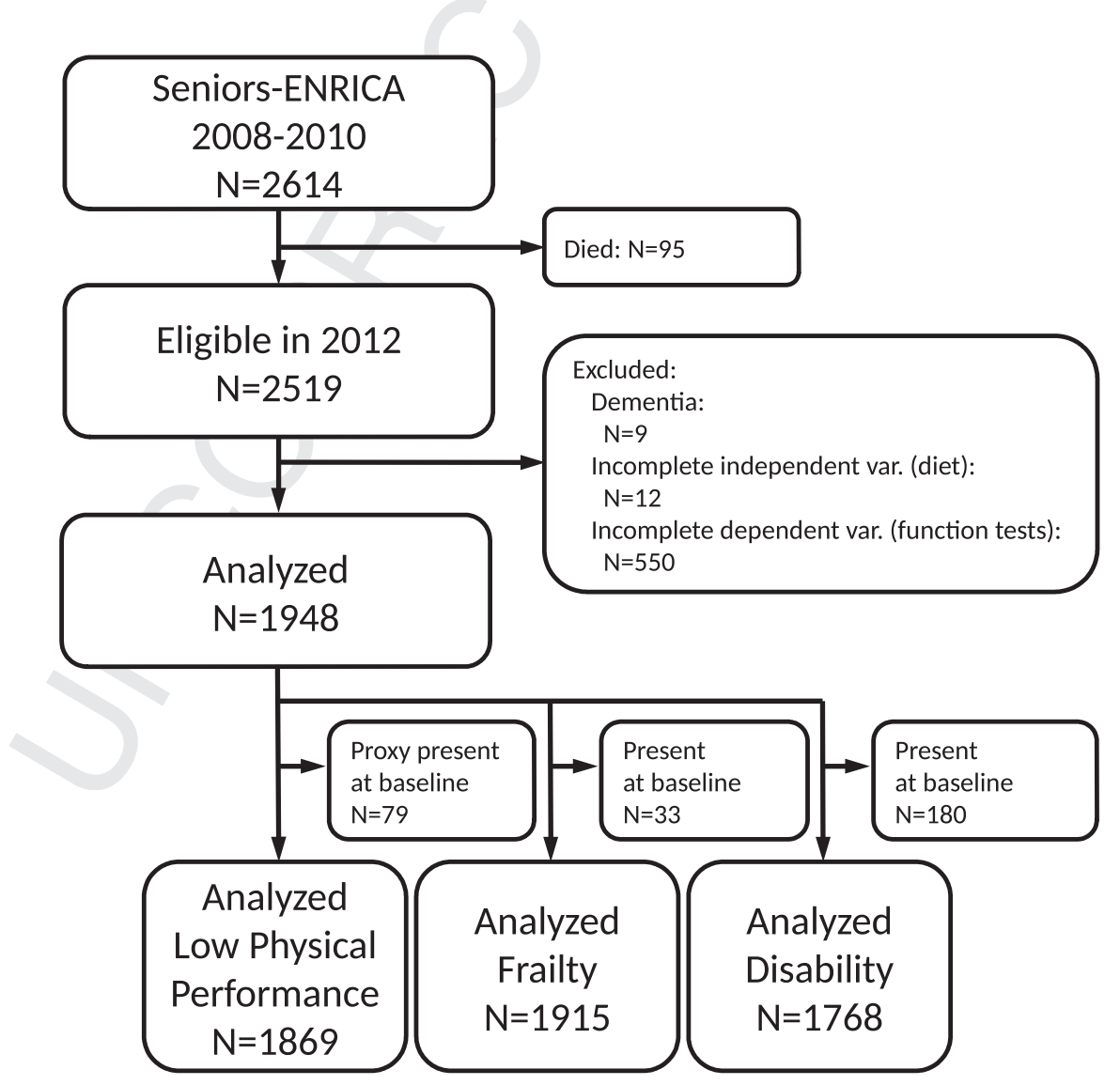

Fig. 1. Flowchart: Steps sequence of participant exclusions to conform the analytic sample. 
size. Group values were multiplied by the group-specific inflammatory coefficient [11] and summed together. Finally, we substracted the mean of these values and divided it by their standard deviation to compute a standardized score. The higher the EDII score the higher the inflammatory component of diet.

\subsection{Outcome measurements: low physical performance, frailty, and disability}

In each participant, to asses a reduced physical performance, we used the Short Physical Performance Battery (SPPB) to evaluate lower extremity function [20]. The SPPB includes three component tests performed in order: 1) Balance: the capacity of holding a standing position with three hierarchical dispositions of feet; 2) Gait speed, across $2.44 \mathrm{~m}$; 3) Chair stand, evaluating the capacity and time needed to rise from a chair five times consecutively without using the arms [20]. A scale between 0 and 4, predefined for each test, is used to score each component and the scores are subsequently summed together (SPPB range $0-12$ ). The standard cut-off score used to detect functional limitation is $\leq 9$ [21]. Additional analyses of each SPPB component were performed using a cut-off $<2$, i.e., scores of 0 or 1 . Such score in any single component implies that the maximum sum could never exceed 9 , i.e., $\mathrm{SPPB} \leq 9$, even with a maximum score, i.e., four, in the other two components.

The presence of three of the following five Fried criteria [22] was used to define frailty: 1) Exhaustion, identified with an affirmative response on any of the two following questions from the Center for Epidemiologic Studies Depression Scale [23]: "I feel that anything I do is a big effort" or "I feel that I cannot keep on doing things" at least 3-4 days a week; 2) Low physical activity, identified when self-reported walking was $\leq 2.5 \mathrm{~h} /$ week in men and $\leq 2 \mathrm{~h} /$ week in women [24]; 3) Slow gait speed, considered as the lowest cohort-specific quintile in the $2.44 \mathrm{~m}$ walking speed test of the SPPB, adjusted for sex and height [20].; 4) Unintentional weight loss, when $\geq 4.5 \mathrm{~kg}$ of body weight was lost in the preceding year; 5) Muscle weakness, when grip strength measured with a Jamar dynamometer (highest of two consecutive measurements in the dominant hand) and adjusted for sex and body mass index (BMI) was in the cohort-specific lowest quintile [25].

Lawton and Brody scale was used to assess disability in instrumental activities of daily living (IADL) [26]. It includes questions about the ability for shopping, phoning, preparing meals, doing housework, laundry, using the transportation, taking medication, and managing finances. Meals and housework questions were only considered for women, because of Spanish cultural issues. A reported difficulty in any of the items classified the participant as having disability.

\subsection{Other variables}

Age, sex, education, smoking status, measured weight and height, from which body mass index (BMI) was calculated, diagnosed diseases, time spent watching TV, and leisure-time physical activity (using the EPIC cohort questionnaire) were collected during the baseline interview and physical exam using standard procedures. Also the MEDAS score [27] assessing conformance of dietary patterns to the Mediterranean diet was calculated.

\subsection{Statistical analyses}

Participants were classified in sex-specific tertiles for each dietary inflammatory index (cut-off values on Table 1 footnote). Logistic regression was used to estimate inter-tertile odds ratios (OR) and their 95\% confidence interval (CI) using the first tertile

Table 1

Characteristics of the study participants across sex-specific tertiles of the Dietary Inflammatory Indices.

\begin{tabular}{|c|c|c|c|c|c|c|c|c|c|}
\hline & \multirow[t]{2}{*}{ Overall } & \multicolumn{3}{|c|}{ Dietary Inflammatory Index (DII) } & \multirow[t]{2}{*}{ P-trend } & \multicolumn{3}{|c|}{ Empirical Dietary Inflammatory Index (EDII) } & \multirow[t]{2}{*}{ P-trend } \\
\hline & & Tertile 1 & Tertile 2 & Tertile 3 & & Tertile 1 & Tertile 2 & Tertile 3 & \\
\hline $\mathrm{N}$ & 1948 & 650 & 648 & 650 & & 650 & 648 & 650 & \\
\hline Men & 48.5 & 48.5 & 48.5 & 48.5 & - & 48.5 & 48.5 & 48.5 & - \\
\hline Age, y & $68.4(6.2)$ & $67.7(5.8)$ & $68.1(6.0)$ & $69.4(6.5)$ & $<0.001$ & $69.1(6.4)$ & $68.3(6.1)$ & $67.8(6.0)$ & $<0.001$ \\
\hline \multicolumn{10}{|l|}{ Education } \\
\hline Primary or less & 52.9 & 49.2 & 50.2 & 59.4 & $<0.001$ & 52.6 & 56.0 & 50.2 & 0.374 \\
\hline Secondary & 24.9 & 28.0 & 26.2 & 20.6 & 0.002 & 27.2 & 22.5 & 25.1 & 0.370 \\
\hline University & 22.1 & 22.8 & 23.6 & 20.0 & 0.229 & 20.2 & 21.5 & 24.8 & 0.045 \\
\hline BMI, $\mathrm{kg} / \mathrm{m}^{2}$ & $28.5(4.3)$ & $28.2(4.3)$ & $28.5(4.1)$ & $28.7(4.5)$ & 0.042 & $28.2(4.0)$ & $28.2(4.2)$ & $29.1(4.6)$ & $<0.001$ \\
\hline Energy intake, kcal/d & $2034.6(572.8)$ & $2285.4(557.7)$ & $2080.6(521.2)$ & $1737.8(499.2)$ & $<0.001$ & $1801.2(507.0)$ & $2001.9(519.0)$ & $2300.5(576.6)$ & $<0.001$ \\
\hline Time spent watching TV,h/wk & $17.7(10.9)$ & $16.9(10.1)$ & $17.2(10.1)$ & $19.2(12.3)$ & $<0.001$ & $17.0(11.0)$ & $17.9(10.7)$ & $18.2(11.0)$ & 0.049 \\
\hline $\begin{array}{l}\text { Leisure-time physical activity, } \\
\text { MET-h/wk }\end{array}$ & $22.0(15.3)$ & $23.1(15.1)$ & $21.7(15.2)$ & $21.1(15.6)$ & 0.021 & $22.4(15.0)$ & $22.4(15.8)$ & $21.1(15.1)$ & 0.118 \\
\hline MEDAS & $7.2(1.8)$ & $8.2(1.8)$ & $6.9(1.8)$ & $6.5(1.5)$ & $<0.001$ & $7.6(1.7)$ & $7.2(1.7)$ & $6.7(2.0)$ & $<0.001$ \\
\hline \multicolumn{10}{|l|}{ Smoking status } \\
\hline Current smoker & 11.4 & 9.1 & 11.7 & 13.5 & 0.012 & 12.5 & 10.8 & 11.1 & 0.433 \\
\hline Former smoker & 30.3 & 30.2 & 33.5 & 27.4 & 0.278 & 30.3 & 31.2 & 29.5 & 0.763 \\
\hline Never smoker & 58.2 & 60.8 & 54.8 & 59.1 & 0.536 & 57.2 & 58.0 & 59.4 & 0.431 \\
\hline \multicolumn{10}{|l|}{ Diagnosed diseases } \\
\hline Diabetes & 15.0 & 12.0 & 14.4 & 18.6 & 0.001 & 12.3 & 13.7 & 18.9 & 0.001 \\
\hline Bronchitis or asthma & 7.3 & 8.0 & 7.4 & 6.6 & 0.339 & 9.2 & 5.4 & 7.4 & 0.203 \\
\hline Cardiovascular disease & 5.1 & 4.6 & 5.9 & 4.9 & 0.802 & 4.5 & 4.9 & 6.0 & 0.210 \\
\hline Osteo-muscular disease & 47.7 & 49.2 & 46.8 & 47.2 & 0.470 & 48.8 & 45.5 & 48.9 & 0.956 \\
\hline Depression & 7.8 & 7.2 & 7.1 & 9.1 & 0.215 & 6.9 & 7.3 & 9.2 & 0.122 \\
\hline Cancer & 2.0 & 1.5 & 2.5 & 2.0 & 0.553 & 2.6 & 1.2 & 2.2 & 0.553 \\
\hline
\end{tabular}

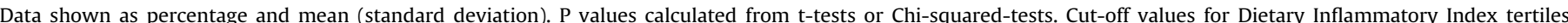

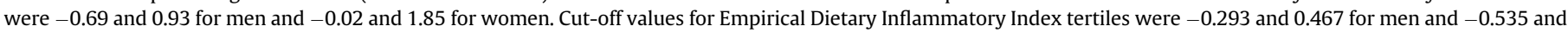
1.220 for women.

BMI, Body Mass Index, MET, metabolic equivalent, MEDAS, MEditerranean Diet Adherence Score (0-14).

Mean (standard deviations) and percentage (number) are reported for continuous and categorical variables respectively. 
(less inflammatory pattern) as reference. A trend significance test was performed considering the tertile assignation as numeric. Regression models were built with three levels of adjustment: Model 1 is adjusted for age, sex, and education; Model 2 is additionally adjusted for smoking status, BMI, energy intake, and diagnosed diseases; Model 3 is additionally adjusted for time spent watching TV and leisure-time physical activity. These were segregated from Model 2 because they can be considered either as confounders or as intermediate variables of the studied associations. Providing Model 2 and 3 separately allows interpreting the results from one or the other approach.

Exclusion of participants exhibiting the target characteristic or a proxy at baseline was done separately for each outcome (see Flowchart), i.e., for analysis of each outcome, participants with it at baseline were considered not at risk and removed. Unfortunately, SPPB was not performed at baseline and participants were excluded from the analyses of incident reduced physical performance (measured at follow-up as low SPPB and low SPPB components) using as a proxy for baseline low physical performance an item from a baseline questionnaire: answering "a lot" to the question "Does your current health or fitness limit you for walking several blocks (a few hundred meters)?". Of these participants excluded for having an affirmative baseline answer to that question, $80 \%$ had low SPPB at follow-up. See the discussion section for an analysis of the consequences of this approximation. Frail participants at baseline were excluded from analyses of the development of frailty outcome. Only robust participants, i.e. without any criteria, were considered at risk for the frailty components analysis. The Lawton and Brody test at baseline was used to exclude participants with any difficulty already at baseline from incident disability analyses. The number at risk for each subsample is reported in the tables and in the Flowchart.

We performed as sensitivity analyses stratification by age (more or less than $70 \mathrm{y}$ ) and by BMI (normal weight, overweight, or obesity).

\section{Results}

At baseline, both inflammatory indices were higher in women, and in those with greater BMI and TV watching time, those who suffered diabetes, and those who were less adherent to Mediterranean dietary patterns. Inflammatory indices differed in the way they were associated with age, level of education, and energy intake; among older, less educated, and people with less caloric diets, DII was higher, but on the contrary, EDII was lower. DII showed higher scores associated with performing less leisure-time physical activity and with being current smoker, but EDII showed no association (Table 1 ).

A low $(\leq 9)$ score in the SPPB at follow-up showed ORs above one for both DII and EDII, but it did not reach statistical significance. Higher DII was associated with increased risk of frailty (OR for the highest vs lowest tertile: $2.48,95 \% \mathrm{CI} 1.42,4.44$, $\mathrm{p}$ for trend $=0.001$ ) and IADL disability (OR: $1.96,95 \%$ CI 1.03, 3.86, p for trend $=0.035$ ) at follow-up, independently of age, sex, education, smoking status, BMI, energy intake, diagnosed diseases, TV watching time, and leisure-time physical activity. However, EDII did not show an association with the risk of frailty or IADL disability (Table 2).

Additionally we examined the association of the DII or EDII with the components of the SPPB and each frailty criterion. Higher scores on both indices were associated with a low score in the gait speed SPPB test; specifically, the OR $(95 \% \mathrm{CI})$ for the highest versus lowest tertile was $1.82(1.27,2.62$, $\mathrm{p}$ for trend $=0.001)$ for DII and 1.39 $(0.99,1.96, \mathrm{p}$ for trend $=0.056)$ for EDII. The frailty criterion that showed a significant association with DII was slowness, using a different threshold that the one used for SPPB scoring, with OR 1.64 $(95 \%$ CI 1.08, 2.51, p-trend $=0.021)$. Unintentional weight loss also showed a tendency to be associated with DII, with OR $1.71(0.97$, 3.03 , p-trend $=0.059$ ), but without reaching the level of significance. For completeness, the associations of frailty components with EDII were studied but, as expected, no association was found, consistently with the absence of a pattern of frailty across EDII tertiles (Appendix Table 1).

Table 2

Odds ratios (95\% Confidence Interval) for the association of Dietary Inflammatory Indices with reduced physical performance, frailty and disability.

\begin{tabular}{|c|c|c|c|c|c|c|c|c|}
\hline & \multicolumn{3}{|c|}{ Dietary Inflammatory Index (DII) } & \multirow[t]{2}{*}{ P-trend } & \multicolumn{3}{|c|}{$\begin{array}{l}\text { Empirical Dietary Inflammatory Index } \\
\text { (EDII) }\end{array}$} & \multirow[t]{2}{*}{ P-trend } \\
\hline & Tertile 1 & Tertile 2 & Tertile 3 & & Tertile 1 & Tertile 2 & Tertile 3 & \\
\hline Low physical performance (SPPB $\leq 9$ ), n/N & $209 / 626$ & $238 / 630$ & $253 / 613$ & $700 / 1869$ & $235 / 626$ & $215 / 620$ & $250 / 623$ & $700 / 1869$ \\
\hline Model 1 & Ref. & $\begin{array}{l}1.18 \\
(0.92,1.50)\end{array}$ & $\begin{array}{l}1.18 \\
(0.93,1.51)\end{array}$ & 0.180 & Ref. & $\begin{array}{l}0.92 \\
(0.72,1.17)\end{array}$ & $\begin{array}{l}1.29 \\
(1.01,1.65)\end{array}$ & 0.040 \\
\hline Model 2 & Ref. & $\begin{array}{l}1.21 \\
(0.94,1.56)\end{array}$ & $\begin{array}{l}1.25 \\
(0.95,1.65)\end{array}$ & 0.113 & Ref. & $\begin{array}{l}0.93 \\
(0.72,1.20)\end{array}$ & $\begin{array}{l}1.18 \\
(0.90,1.55)\end{array}$ & 0.243 \\
\hline Model 3 & Ref. & $\begin{array}{l}1.19 \\
(0.92,1.53)\end{array}$ & $\begin{array}{l}1.22 \\
(0.92,1.62)\end{array}$ & 0.159 & Ref. & $\begin{array}{l}0.94 \\
(0.73,1.21)\end{array}$ & $\begin{array}{l}1.18 \\
(0.90,1.55)\end{array}$ & 0.240 \\
\hline Frailty, n/N & $25 / 641$ & $35 / 640$ & $67 / 634$ & $127 / 1915$ & $46 / 640$ & $37 / 637$ & $44 / 638$ & $127 / 1915$ \\
\hline Model 1 & Ref. & $\begin{array}{l}1.33 \\
(0.78,2.30)\end{array}$ & $\begin{array}{l}2.30 \\
(1.43,3.80)\end{array}$ & $<0.001$ & Ref. & $\begin{array}{l}0.82 \\
(0.52,1.31)\end{array}$ & $\begin{array}{l}1.10 \\
(0.71,1.73)\end{array}$ & 0.681 \\
\hline Model 2 & Ref. & $\begin{array}{l}1.32 \\
(0.76,2.34)\end{array}$ & $\begin{array}{l}2.60 \\
(1.50,4.61)\end{array}$ & $<0.001$ & Ref. & $\begin{array}{l}0.85 \\
(0.52,1.38)\end{array}$ & $\begin{array}{l}0.84 \\
(0.50,1.41)\end{array}$ & 0.502 \\
\hline Model 3 & Ref. & $\begin{array}{l}1.33 \\
(0.75,2.37)\end{array}$ & $\begin{array}{l}2.48 \\
(1.42,4.44)\end{array}$ & 0.001 & Ref. & $\begin{array}{l}0.82 \\
(0.50,1.34)\end{array}$ & $\begin{array}{l}0.77 \\
(0.45,1.30)\end{array}$ & 0.319 \\
\hline IADL disability, $\mathrm{n} / \mathrm{N}$ & $17 / 605$ & $22 / 591$ & $42 / 572$ & $81 / 1768$ & $32 / 602$ & $23 / 586$ & $26 / 580$ & $81 / 1768$ \\
\hline Model 1 & Ref. & $\begin{array}{l}1.26 \\
(0.66,2.45)\end{array}$ & $\begin{array}{l}2.21 \\
(1.24,4.07)\end{array}$ & 0.005 & Ref. & $\begin{array}{l}0.79 \\
(0.45,1.38)\end{array}$ & $\begin{array}{l}1.01 \\
(0.58,1.75)\end{array}$ & 0.999 \\
\hline Model 2 & Ref. & $\begin{array}{l}1.22 \\
(0.62,2.41)\end{array}$ & $\begin{array}{l}2.03 \\
(1.07,3.99)\end{array}$ & 0.026 & Ref. & $\begin{array}{l}0.88 \\
(0.49,1.56)\end{array}$ & $\begin{array}{l}1.14 \\
(0.61,2.10)\end{array}$ & 0.734 \\
\hline Model 3 & Ref. & $\begin{array}{l}1.20 \\
(0.62,2.38)\end{array}$ & $\begin{array}{l}1.96 \\
(1.03,3.86)\end{array}$ & 0.035 & Ref. & $\begin{array}{l}0.87 \\
(0.48,1.56)\end{array}$ & $\begin{array}{l}1.10 \\
(0.59,2.05)\end{array}$ & 0.796 \\
\hline
\end{tabular}

$\mathrm{n} / \mathrm{N}$, number of cases/number at risk. SPPB, short physical performance battery. IADL, instrumental activities of daily living.

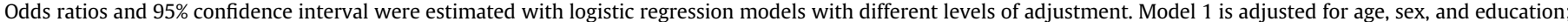

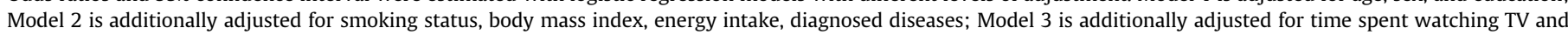
leisure-time physical activity. Trend is calculated with the tertile number as a continuous variable. 
Similar results were observed when analyses were stratified by age or BMI categories (data not shown).

\section{Discussion}

In this cohort of older adults followed up for 3 years, DII at baseline was associated with later presence of slow walking speed. DII was associated with development of frailty and of a positive Lawton and Brody test, indicating IADL disability.

Diet has been linked with both frailty $[6,7,28]$ and inflammatory markers [10,29]. Nutritionally deficient diets, on the one hand, may accelerate musculoskeletal decline through compromising protein synthesis, producing sarcopenia, and loss of muscle strength. On the other hand, obesity is a condition that predicts future frailty $[30,31]$, probably favoring low physical activity and fatigue. Interestingly, not only specific deficiencies or obesogenic diets affect future frailty; dietary patterns recognized for providing protective health effects for several chronic diseases, such as the Mediterranean diet, also confer protection against incident frailty [6].

Regular intake of several individual foods and nutrients, like vitamins, antioxidants, n-3 fatty acids, and alcohol, modify inflammatory markers [10]. Also the Mediterranean dietary pattern is associated with reduced markers of inflammation [29]. Based on published evidence, Shivappa et al. [10] developed DII, an a priori dietary pattern that provides a quantitative assessment of the inflammatory potential of a particular diet. We found that Shivappa's index predicts incident frailty and disability for IADL after adjustment for confounders. A second index, EDII, has been developed as an a posteriori dietary pattern applying reduced rank regression to Nurses' Health Study data [11]. The selection of foods and their weighting in this index were tuned to capture cytokine levels in that sample. EDII was not linked to the reduced physical performance, frailty, or disability outcomes.

In our data DII and EDII also showed differing association with socio-demographics, lifestyle, and other potential confounders, suggesting that they capture different information from the analyzed diets. Our aim was to gather information on dietary patterns that may affect healthy aging and to identify which index of dietary inflammatory potential does capture better information of a relevant pattern. Our goal was not to compare the indices in their capacity for detecting inflammation, which should be addressed with other methods, including biological assessment of inflammation. We propose some explanations for why association of the indices with frailty differ. The indices are different in development, structure, and potential uses, which could explain the distinct results, at least partially. DII is an index based mainly on nutrients, minerals, and some condiments (usually consumed in small quantities and supplying a minimum amount of energy), and as such, it requires more details in data collection and availability of appropriate food composition tables. Due to the high complexity of nutrient-based indices, they are used mainly in research. Nonetheless, given that most nutrients and minerals considered are usually present in many foods, when those nutrients are active principles, nutrient-based indices are able to capture better the hidden relationship between diets and outcomes because they take into account elementary components from the entire diet. In addition, DII is based in previous knowledge of associations and their possible physiological pathways, and each component of the index has already received attention in trying to explain the biological plausibility of its association with inflammation. On the contrary, EDII is a food-based index, in particular based on food groups. Indices of this kind are more suitable for clinical use (screening, risk communication, and interventions) but they are more difficult to be applied across countries and cultures because food components and their relevance in the diet may vary substantially. Also, they are usually less sensitive to small effects of active principles because foods, and food groups, may contain antagonistic principles that partially neutralize each other. EDII was derived statistically, and a common criticism on a posteriori indices is that they underperform when ported from the population in which they were derived to other populations. Furthermore, healthy or unhealthy aging is likely to depend on nutrients that act as active principles, thus DII seems to capture better the underlying association than EDII in which only food groups are considered.

Among the theories addressing frailty pathogenesis, chronic inflammation has been proposed as a key underlying mechanism [32]. Several studies have shown increased levels of interleukin-6 (IL-6) [33], C-reactive protein [34], and leukocytes [33] among frail community-dwellers, as well as among frail residents in nursing homes and assisted living facilities [35]. Thus, frailty could be considered an inflammatory status. In the search for a pathophysiological explanation, proposed intermediate links between chronic inflammation and frailty are muscular wasting, producing sarcopenia, endocrine dysregulation including cortisol and the somatotropin axes, cardiovascular diseases, and nutritional dysregulation with deficiency of micronutrients [32]. Nonetheless, these theories have not been proven so far, and a recent meta-analysis of studies on the prospective association between baseline C-reactive protein or interleukin- 6 and frailty rendered non-significant results after combining data of 3402 participants with a median follow-up of 3 years [9]. Our results, in which inflammatory dietary patterns significantly predict incident frailty, support the pathogenic implication of inflammatory mechanisms in frailty.

In order to obtain a deeper insight of the aspects of physical decline which are most affected by the inflammatory dietary patterns, we analyzed the components of the SPPB and frailty. We found a significant association of DII with slow gait speed (which is a component of SPPB and frailty, with different thresholds), and borderline significant association of EDII with the gait speed component of the SPPB. Gait speed is the objective physical performance test most associated with frailty diagnosis and it predicts most endpoints that frailty predisposes to [36]: death, falls, disability, and hospitalization. Slow gait alone is associated with a pro-inflammatory pattern of cytokines [37], but a study of inflammatory and walking speed trajectories did not find evidence of any interactive effect of them on mortality [38]. Again, cross-sectional evidence of association between inflammation and gait speed is clear [39], but longitudinal evidence suggests that a common cause, such as adiposity could be responsible of the joint development of both [40]. We found a statistically significant prospective association between inflammatory potential of diet and slowness. The kind of diet represented by the DII and EDII could be responsible of producing both effects: activating pro-inflammatory mechanisms and producing physical decline. In fact, both DII and EDII were inversely associated with MEDAS, which measures adherence to a Mediterranean diet, which is considered to be healthy and, as mentioned before, associated with lower inflammation [29] and decreased frailty risk [6].

This study benefits from a prospective design, a detailed measurement of diet with a validated instrument, as well as a reasonably large sample size. Outcome measurements have been collected by trained staff undergoing a strict re-training schedule to ensure homogeneity and objectivity. There are also some limitations in our analyses. First, only 32 of 45 Shivappa's items were available in our dietary data. Fortunately, DII was conceived to be calculated with the amount of items available in each research context and two thirds is around the fraction regularly used in other studies. Second, applying a posteriori patterns (EDII) to populations other than that where they were developed can bias associations towards the null. Third, differences in habitual diets across countries may create 
heterogeneity on the foods providing the inflammatory or antiinflammatory effects; also some associations of the inflammatory dietary indices with socio-demographic and lifestyle variables may vary across populations. In our analyses, adjusting for these variables was used to control confounding but also to tackle with those differences; however, we cannot rule out some residual confounding. The lack of SPPB at baseline implies an incomplete exclusion of low SPPB prevalent cases for the analysis. This could artificially show a spurious longitudinal association due to contamination from cross-sectional association but given that we do not find such longitudinal association, even with the potential contamination, we believe that the approximation does not affect the results reported.

In conclusion, the different dietary inflammatory indices seem to capture non-overlapping information on the inflammatory characteristics of diet. Higher inflammatory potential of diet measured by DII but not EDII is associated with future frailty and IADL disability. Our results provide additional evidence for the role of inflammation on frailty, and shows that DII is able to measure diet healthiness in terms of physical decline in addition to avoidance of inflammation.

\section{Q5 $\quad$ Funding}

This work was supported by FIS grants 16/609, 14/00009, and 13/0288 (Instituto de Salud Carlos III, State Secretary of R+D+I, and co-funded by European Regional Development Fund/European Social Fund "Investing in your future"), the FRAILOMIC Initiative (FP7-HEALTH-2012-Proposal no. 305483-2), the ATHLOS project (EU H2020- Project ID: 635316) and the JPI HDHL (SALAMANDER Q6 project).

\section{Conflict of interest}

The authors have no potential conflicts of interest.

\section{Acknowledgements}

Dr. Laclaustra's research activity is funded by Agencia Aragonesa para la Investigación y el Desarrollo (ARAID). This work was supported by FIS grants 16/609, 14/00009, and 13/0288 (Instituto de Salud Carlos III, State Secretary of R+D+I, and co-funded by European Regional Development Fund/European Social Fund "Investing in your future"), the FRAILOMIC Initiative (FP7-HEALTH-2012Proposal no. 305483-2), the ATHLOS project (EU H2020- Project ID: 635316) and the JPI HDHL (SALAMANDER project). The funding agencies had no role in the study design, data analysis, interpretation of results, manuscript preparation, or in the decision to submit this manuscript for publication.

ML, FRA, and ELG designed research; ML conducted research; PGC, JRB, AG, EGE, and ELG collected data; FRA and JRB designed the initial cohort; ML and ELG analyzed data; ML, FRA, and ELG drafted the manuscript; The rest of the authors contributed important intellectual content to the final paper; ML had primary responsibility for final content. All authors read and approved the final manuscript.

\section{Appendix A. Supplementary data}

Supplementary data to this chapter can be found online at https://doi.org/10.1016/j.clnu.2019.01.013.

\section{References}

[1] Vermeulen J, Neyens JCL, van Rossum E, Spreeuwenberg MD, de Witte LP. Predicting ADL disability in community-dwelling elderly people using physical frailty indicators: a systematic review. BMC Geriatr 2011;11:33. https:// doi.org/10.1186/1471-2318-11-33.

[2] Fried LP, Ferrucci L, Darer J, Williamson JD, Anderson G. Untangling the concepts of disability, frailty, and comorbidity: implications for improved targeting and care. J Gerontol A Biol Sci Med Sci 2004;59:255-63.

[3] World Health Organization, editor. World report on ageing and health. Geneva: WHO; 2015.

[4] Yannakoulia M, Ntanasi E, Anastasiou CA, Scarmeas N. Frailty and nutrition: from epidemiological and clinical evidence to potential mechanisms. Metab Clin Exp 2017;68:64-76. https://doi.org/10.1016/j.metabol.2016.12.005.

[5] Lana A, Rodriguez-Artalejo F, Lopez-Garcia E. Dairy consumption and risk of frailty in older adults: a prospective cohort study. J Am Geriatr Soc 2015;63: 1852-60. https://doi.org/10.1111/jgs.13626.

[6] León-Muñoz LM, Guallar-Castillón P, López-García E, Rodríguez-Artalejo F. Mediterranean diet and risk of frailty in community-dwelling older adults. J Am Med Dir Assoc 2014;15:899-903. https://doi.org/10.1016/ j.jamda.2014.06.013.

[7] León-Muñoz LM, García-Esquinas E, López-García E, Banegas JR, RodríguezArtalejo F. Major dietary patterns and risk of frailty in older adults: a prospective cohort study. BMC Med 2015;13:11. https://doi.org/10.1186/s12916014-0255-6.

[8] Struijk EA, Guallar-Castillón P, Rodríguez-Artalejo F, López-García E. Mediterranean dietary patterns and impaired physical function in older adults. J Gerontol A Biol Sci Med Sci 2016. https://doi.org/10.1093/gerona/glw208.

[9] Soysal P, Stubbs B, Lucato P, Luchini C, Solmi M, Peluso R, et al. Inflammation and frailty in the elderly: a systematic review and meta-analysis. Ageing Res Rev 2016;31:1-8. https://doi.org/10.1016/j.arr.2016.08.006.

[10] Shivappa N, Steck SE, Hurley TG, Hussey JR, Hébert JR. Designing and developing a literature-derived, population-based dietary inflammatory index. Publ Health Nutr 2014;17:1689-96. https://doi.org/10.1017/S1368980 013002115.

[11] Tabung FK, Smith-Warner SA, Chavarro JE, Wu K, Fuchs CS, Hu FB, et al Development and validation of an empirical dietary inflammatory index. J Nutr 2016;146:1560-70. https://doi.org/10.3945/jn.115.228718.

[12] Bodén S, Wennberg M, Van Guelpen B, Johansson I, Lindahl B, Andersson J, et al. Dietary inflammatory index and risk of first myocardial infarction; a prospective population-based study. Nutr J 2017;16:21. https://doi.org/ 10.1186/s12937-017-0243-8.

[13] Garcia-Arellano A, Ramallal R, Ruiz-Canela M, Salas-Salvadó J, Corella D Shivappa N, et al. Dietary inflammatory index and incidence of cardiovascular disease in the PREDIMED study. Nutrients 2015;7:4124-38. https://doi.org 10.3390/nu7064124.

[14] Harmon BE, Wirth MD, Boushey CJ, Wilkens LR, Draluck E, Shivappa N, et al. The dietary inflammatory index is associated with colorectal cancer risk in the multiethnic cohort. J Nutr 2017;147:430-8. https://doi.org/10.3945/ jn.116.242529.

[15] Ruiz-Canela M, Bes-Rastrollo M, Martínez-González MA. The role of dietary inflammatory index in cardiovascular disease, metabolic syndrome and mortality. Int J Mol Sci 2016;17. https://doi.org/10.3390/ijms17081265.

[16] Adjibade M, Andreeva VA, Lemogne C, Touvier M, Shivappa N, Hébert JR, et al. The inflammatory potential of the diet is associated with depressive symptoms in different subgroups of the general population. J Nutr 2017. https:// doi.org/10.3945/in.116.245167.

[17] Shivappa N, Stubbs B, Hébert JR, Cesari M, Schofield P, Soysal P, et al. The relationship between the dietary inflammatory index and incident frailty: a longitudinal cohort study. J Am Med Dir Assoc 2017. https://doi.org/10.1016/ j.jamda.2017.08.006.

[18] Rodríguez-Artalejo F, Graciani A, Guallar-Castillón P, León-Muñoz LM, Zuluaga MC, López-García E, et al. Rationale and methods of the study on nutrition and cardiovascular risk in Spain (ENRICA). Rev Esp Cardiol 2011;64: 876-82. https://doi.org/10.1016/j.recesp.2011.05.019.

[19] Guallar-Castillón P, Sagardui-Villamor J, Balboa-Castillo T, Sala-Vila A, Ariza Astolfi MJ, Sarrión Pelous MD, et al. Validity and reproducibility of a Spanish dietary history. PLoS One 2014;9, e86074. https://doi.org/10.1371/ journal.pone.0086074.

[20] Guralnik JM, Simonsick EM, Ferrucci L, Glynn RJ, Berkman LF, Blazer DG, et al. A short physical performance battery assessing lower extremity function: association with self-reported disability and prediction of mortality and nursing home admission. J Gerontol 1994;49:M85-94.

[21] Guralnik JM, Ferrucci L, Pieper CF, Leveille SG, Markides KS, Ostir GV, et al. Lower extremity function and subsequent disability: consistency across studies, predictive models, and value of gait speed alone compared with the short physical performance battery. J Gerontol A Biol Sci Med Sci 2000;55: M221-31.

[22] Fried LP, Tangen CM, Walston J, Newman AB, Hirsch C, Gottdiener J, et al. Frailty in older adults: evidence for a phenotype. J Gerontol A Biol Sci Med Sc 2001;56:M146-56.

[23] Radloff LS. The CES-D scale: a self-report depression scale for research in the general population. Appl Psychol Meas 1977;1:385-401. https://doi.org/ 10.1177/014662167700100306. 
[24] Washburn RA, Smith KW, Jette AM, Janney CA. The physical activity scale for the elderly (PASE): development and evaluation. J Clin Epidemiol 1993;46: 153-62.

[25] Ottenbacher KJ, Branch LG, Ray L, Gonzales VA, Peek MK, Hinman MR. The reliability of upper- and lower-extremity strength testing in a community survey of older adults. Arch Phys Med Rehabil 2002;83:1423-7.

[26] Lawton MP, Brody EM. Assessment of older people: self-maintaining and instrumental activities of daily living. Gerontologist 1969;9:179-86.

[27] Schröder H, Fitó M, Estruch R, Martínez-González MA, Corella D, SalasSalvadó J, et al. A short screener is valid for assessing Mediterranean diet adherence among older Spanish men and women. J Nutr 2011;141:1140-5. https://doi.org/10.3945/jn.110.135566.

[28] Pilleron S, Ajana S, Jutand M-A, Helmer C, Dartigues J-F, Samieri C, et al. Dietary patterns and 12-year risk of frailty: results from the three-city bordeaux study. J Am Med Dir Assoc 2017;18:169-75. https://doi.org/10.1016/ j.jamda.2016.09.014.

[29] Lopez-Garcia E, Schulze MB, Fung TT, Meigs JB, Rifai N, Manson JE, et al. Major dietary patterns are related to plasma concentrations of markers of inflammation and endothelial dysfunction. Am J Clin Nutr 2004;80:1029-35.

[30] Strandberg TE, Sirola J, Pitkälä KH, Tilvis RS, Strandberg AY, Stenholm S. Association of midlife obesity and cardiovascular risk with old age frailty: a 26year follow-up of initially healthy men. Int J Obes (Lond) 2012;36:1153-7 https://doi.org/10.1038/ijo.2012.83.

[31] García-Esquinas E, José García-García F, León-Muñoz LM, Carnicero JA, Guallar-Castillón P, Gonzalez-Colaço Harmand M, et al. Obesity, fat distribution, and risk of frailty in two population-based cohorts of older adults in Spain. Obesity (Silver Spring) 2015;23:847-55. https://doi.org/10.1002/oby.21013.

[32] Chen X, Mao G, Leng SX. Frailty syndrome: an overview. Clin Interv Aging 2014;9:433-41. https://doi.org/10.2147/CIA.S45300.
[33] Leng SX, Xue Q-L, Tian J, Walston JD, Fried LP. Inflammation and frailty in older women. J Am Geriatr Soc 2007;55:864-71. https://doi.org/10.1111/ j.1532-5415.2007.01186.x.

[34] Hubbard RE, O'Mahony MS, Savva GM, Calver BL, Woodhouse KW. Inflammation and frailty measures in older people. J Cell Mol Med 2009;13:3103-9. https://doi.org/10.1111/j.1582-4934.2009.00733.x.

[35] Langmann GA, Perera S, Ferchak MA, Nace DA, Resnick NM, Greenspan SL. Inflammatory markers and frailty in long-term care residents. J Am Geriatr Soc 2017. https://doi.org/10.1111/jgs.14876.

[36] Pamoukdjian F, Paillaud E, Zelek L, Laurent M, Lévy V, Landre T, et al. Measurement of gait speed in older adults to identify complications associated with frailty: a systematic review. J Geriatr Oncol 2015;6:484-96. https:// doi.org/10.1016/j.jgo.2015.08.006.

[37] Marzetti E, Landi F, Marini F, Cesari M, Buford TW, Manini TM, et al. Patterns of circulating inflammatory biomarkers in older persons with varying levels of physical performance: a partial least squares-discriminant analysis approach. Front Med (Lausanne) 2014;1:27. https://doi.org/10.3389/fmed.2014.00027.

[38] Brown PJ, Roose SP, Zhang J, Wall M, Rutherford BR, Ayonayon HN, et al. Inflammation, depression, and slow gait: a high mortality phenotype in later life. J Gerontol A Biol Sci Med Sci 2016;71:221-7. https://doi.org/10.1093/ gerona/glv156.

[39] Windham BG, Wilkening SR, Lirette ST, Kullo IJ, Turner ST, Griswold ME, et al. Associations between inflammation and physical function in African Americans and European Americans with prevalent cardiovascular risk factors. J Am Geriatr Soc 2016;64:1448-55. https://doi.org/10.1111/jgs.14229.

[40] Beavers KM, Hsu F-C, Houston DK, Beavers DP, Harris TB, Hue TF, et al. The role of metabolic syndrome, adiposity, and inflammation in physical performance in the Health ABC Study. J Gerontol A Biol Sci Med Sci 2013;68: 617-23. https://doi.org/10.1093/gerona/gls213. 